\title{
Covalently Functionalized Nanotubes as Nanometer-Sized Probes in Chemistry and Biology
}

\section{Citation}

Wong, Stanislaus S., Ernesto Joselevich, Adam T. Woolley, Chin Li Cheung, and Charles M. Lieber. 1998. Covalently functionalized nanotubes as nanometer-sized probes in chemistry and biology. Nature 394: 52-55.

\section{Published Version}

http://dx.doi.org/10.1038/27873

\section{Permanent link}

http://nrs.harvard.edu/urn-3:HUL.InstRepos:2710385

\section{Terms of Use}

This article was downloaded from Harvard University's DASH repository, and is made available under the terms and conditions applicable to Other Posted Material, as set forth at http:// nrs.harvard.edu/urn-3:HUL.InstRepos:dash.current.terms-of-use\#LAA

\section{Share Your Story}

The Harvard community has made this article openly available.

Please share how this access benefits you. Submit a story.

\section{Accessibility}




\title{
Covalently Functionalized Nanotubes as Nanometer-Sized Probes in Chemistry and Biology
}

\author{
Stanislaus S. Wong, Ernesto Joselevich, Adam T. Woolley, Chin Li Cheung, \\ and Charles M. Lieber
}

Department of Chemistry and Chemical Biology, Harvard University, 12 Oxford Street, Cambridge, Massachusetts 02138, USA Correspondence: Charles M. Lieber (email cml@cmliris.harvard.edu).

\begin{abstract}
Carbon nanotubes combine a range of properties that make them well suited for use as probe tips in applications such as atomic force microscopy $(\mathrm{AFM})^{1,2,3}$. Their high aspect ratio, for example, opens up the possibility of probing the deep crevices ${ }^{4}$ that occur in microelectronic circuits, and the small effective radius of nanotube tips significantly improves the lateral resolution beyond what can be achieved using commercial silicon tips ${ }^{5}$. Another characteristic feature of nanotubes is their ability to buckle elastically ${ }^{4},{ }^{6}$, which makes them very robust while limiting the maximum force that is applied to delicate organic and biological samples. Earlier investigations into the performance of nanotubes as scanning probe microscopy tips have focused on topographical imaging, but a potentially more significant issue is the question of whether nanotubes can be modified to create probes that can sense and manipulate matter at the molecular level ${ }^{7}$. Here we demonstrate that nanotube tips with the capability of chemical and biological discrimination can be created with acidic functionality and by coupling basic or hydrophobic functionalities or biomolecular probes to the carboxyl groups that are present at the open tip ends. We have used these modified nanotubes as AFM tips to titrate the acid and base groups, to image patterned samples based on molecular interactions, and to measure the binding force between single protein-ligand pairs. As carboxyl groups are readily derivatized by a variety of reactions ${ }^{8}$, the preparation of a wide range of functionalized nanotube tips should be possible, thus creating molecular probes with potential applications in many areas of chemistry and biology.
\end{abstract}

Among the many reactions that can be used to derivatize carboxy $(-\mathrm{COOH})$ functional groups, we have concentrated on nanotube chemical modification that involves the coupling of amines to thecarboxyl groups at the tip ends to form amide-linked groups (Figure 1a) ${ }^{9}$. The broad applicability of this coupling reaction to aqueous and non-aqueous chemistry makes it especially attractive for nanotube functionalization.

Open-ended nanotube tips are formed while shortening the tubes in an oxidizing environment before use. A transmission electron microscopy (TEM) image of a multi-walled nanotube tip end demonstrates that this process produces open ends (Figure 1a, inset). Carboxyl groups are expected at these open ends on the basis of previous spectroscopic studies of oxidized bulk nanotube ${ }^{10}$ and graphite ${ }^{11},{ }^{12}$ samples. Such traditional analytical techniques are nevertheless limited in their ability to verify directly the functional groups at the very end of a specific tip, because in the ideal limit there will be only a single such group $\left(\sim 10^{-24} \mathrm{~mol}\right)$.

An alternative approach for assessing the functionality at a specific nanotube tip end is to measure the adhesion force between the tip and a surface that terminates in a known chemical functionality (that is, chemical force microscopy) ${ }^{13,14,15}$. Previous adhesion measurements carried out as a function of solution $\mathrm{pH}$ (force titrations) between gold-coated $\mathrm{Si}_{3} \mathrm{~N}_{4}$ tips and substrates functionalized with selfassembled monolayers (SAMs) terminating in carboxyl and hydroxyl groups showed that the fraction of proton dissociation from the surface carboxyl groups could be readily monitored by the drop in adhesion force as in a classic $\mathrm{pH}$ titration ${ }^{14},{ }^{16},{ }^{17}$. If carboxyl groups do indeed exist at the nanotube ends, then they also should be able to be titrated in the same way. Force titrations between $\mathrm{pH} 2$ and 9 with multi-walled nanotube tips on hydroxyl-terminated SAM substrates show a well defined drop in the adhesion force at $\mathrm{pH} \sim 4.5$ (Figure 1b) that is characteristic of the deprotonation of a carboxylic acid; the mid-point of this drop (4.5) is assigned to be the $\mathrm{p} K_{\mathrm{a}}$. In these and all other experiments described below, the applied loads were kept below the force required for nanotube buckling ${ }^{4},{ }^{5}$ to ensure that only the nanotube end contacted the surface. The observed decrease in adhesion force with increasing $\mathrm{pH}$ is also reversible for a given tip, and the transition is observed reproducibly for other tips. The absolute value of the adhesion force at low $\mathrm{pH}$ can vary between tips, and we believe that this reflects a variation in the number of carboxyl groups exposed at the ends of different tips. Last, the similarity of the value of the $\mathrm{p} K_{\mathrm{a}}$ determined in our force titrations (4.5) to the bulk solution value for benzoic acid (4.2) implies that the carboxyl group is well solvated and accessible to reaction ${ }^{16}$.

To investigate the covalent modification of nanotube tips we have coupled amines $\left(\mathrm{RNH}_{2}\right)$, which yield non-ionizable or ionizable functionalities on the tips, using carbodiimide chemistry that selectively forms amide linkages only with carboxyl groups (Figure 1a) $)^{9},{ }^{18}$. Nanotube tips modified with benzylamine - which should expose non-ionizable, hydrophobic functional groups at the tip end - yield the expected $\mathrm{pH}$-independent interaction force on hydroxyl-terminated SAM substrates. This covalent modification thus eliminates the prominent $\mathrm{pH}$-dependent behaviour observed with the unfunctionalized tips. Moreover, force titrations with ethylenediamine modified (that is, amine-functionalized) tips show no adhesion at low $\mathrm{pH}$ and finite adhesion above $\mathrm{pH} 7$. These $\mathrm{pH}$-dependent interactions are con- 
sistent with our expectations for an exposed basic amine functionality that is protonated and charged at low $\mathrm{pH}$ and neutral at high $\mathrm{pH}$. Although the observed $\mathrm{p} K_{\mathrm{a}}$ of the nanotube-bound amine $(\sim 7)$ is reduced relative to homogeneous solution (9-10), similar behaviour has been observed in previous studies of SAM-modified $\mathrm{Si}_{3} \mathrm{~N}_{4}$ tips $^{16}$. Additional experiments carried out on independent tips modified using
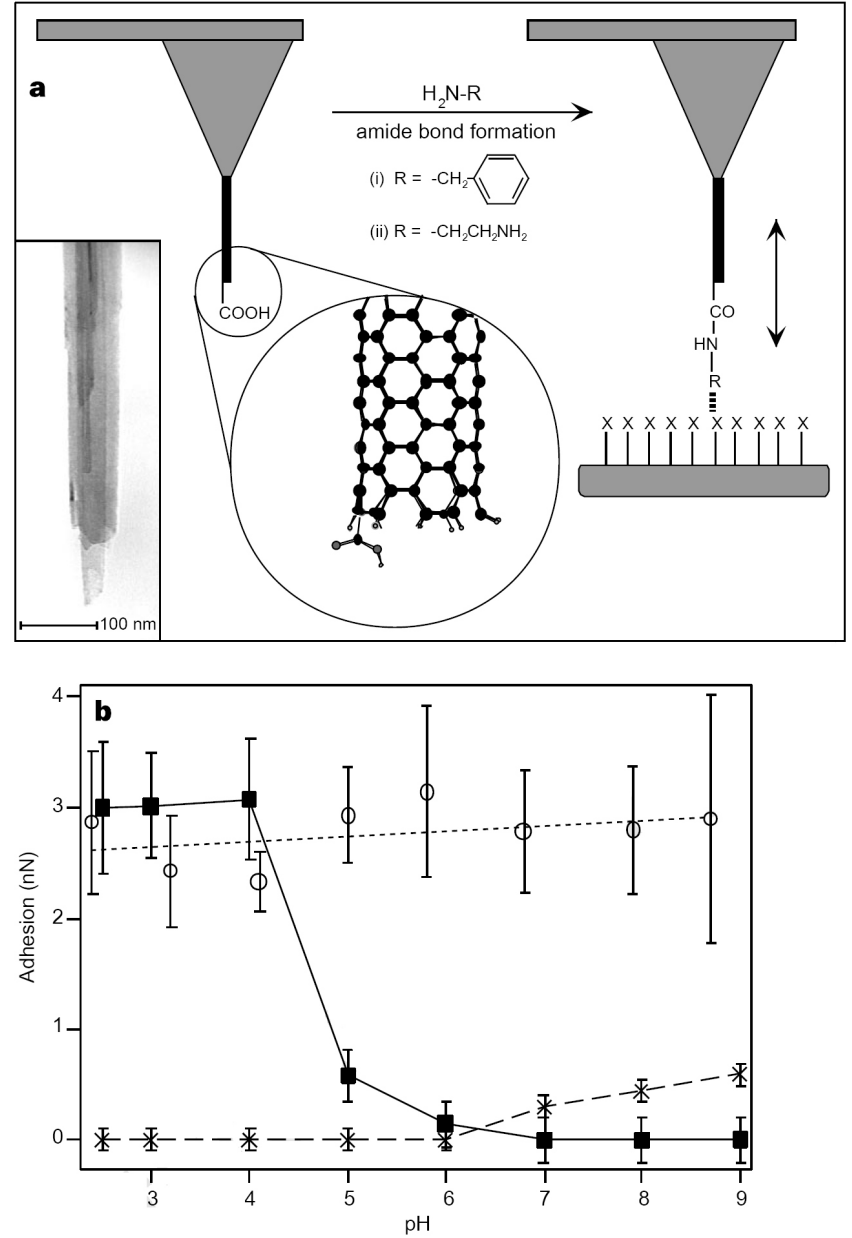

Figure 1. Preparation and characterization of functionalized carbon nanotube tips. a, Diagram illustrating the modification of a nanotube tip by coupling an amine $\left(\mathrm{RNH}_{2}\right)$ to a pendant carboxyl group, and the application of this probe to sense specific interactions with functional groups (X) of a substrate. The circular inset is a molecular model of a single nanotube wall with one carboxyl group at the tip end. The multiwalled nanotubes used in our studies were attached to the pyramids of gold-coated Si cantilevers ( $k=0.5-5 \mathrm{~N} \mathrm{~m}^{-1}$, Digital Instruments, Inc.) using an acrylic adhesive under the direct view of an optical microscope $^{5}$. The as-made nanotube tips were shortened by applying a bias voltage between the tip and a sputtered $\mathrm{Nb}$ surface in an oxygen environment. Chemical modification of the nanotube ends was then carried out by placing a cantilever-tip assembly in a solution of $50 \mathrm{mM}$ EDC (1-ethyl-3-(3-dimethylaminopropyl) carbodiimide hydrochloride) (Pierce) and $5 \mathrm{mM}$ of either benzylamine or ethylenediamine in $0.1 \mathrm{M}$ MES (2-[N-morpholino]ethanesulphonic acid) (Sigma) buffer pH 6.0, for 2 hours. The tips were then successively washed in $0.1 \mathrm{M}$ solutions of $\mathrm{MES}, \mathrm{NaCl}$ (Fisher) and deionized water. The nanotubes were prepared by arc-discharge and purified by oxidation $\left(700{ }^{\circ} \mathrm{C}\right.$, air) until $\sim 2 \%$ of the original mass remained. Inset, TEM image showing the open end of a shortened nanotube tip. b. Adhesion force as a function of $\mathrm{pH}$ between the nanotube tips and a hydroxy-terminated SAM (11-thioundecanol on gold-coated mica): filled squares, carboxyl (unmodified); open circles, phenyl (modified with benzylamine); and crosses, amine (modified with ethylenediamine). Each data point corresponds to the mean of 50-100 adhesion measurements, and the error bars represent one standard deviation. benzylamine and ethylenediamine confirm the reproducibility of these results. We believe these data thus demonstrate unambiguously that carboxyl groups are exposed at the ends of nanotube tips, and that these groups can be covalently modified to produce probes with very distinct chemical functionalities.

We have explored several areas where these tips, and our newly developed approach to covalent modification, can be applied. First, the use of functionalized nanotube probes for chemically sensitive imaging has been investigated using patterned SAM substrates (Figure 2). We recorded intermittent-contact or tapping-mode images (Figure 2b) in ethanol solution, using carboxyl-terminated nanotube tips on substrates patterned ${ }^{19}$ with squares that terminate in $\mathrm{CH}_{3}$ groups and surrounded by $\mathrm{COOH}$-terminated regions. The images show a difference in phase between the two sample areas, although there is no difference in height: the tip- $\mathrm{COOH} / \mathrm{sample}-\mathrm{COOH}$ regions show a phase lag relative to the tip- $\mathrm{COOH} / \mathrm{sample}-\mathrm{CH}_{3}$ regions. Recent tappingmode studies using Au-coated $\mathrm{Si}_{3} \mathrm{~N}_{4}$ tips functionalized with SAMs have shown that phase-lag differences can be quantitatively related to differences in the adhesion forces, and thus can be interpreted in terms of a map of the chemical functionality ${ }^{20}$. Because we expect ${ }^{13}$ (and indeed find) the adhesion force between the carboxyl-terminated nanotube tip and the $\mathrm{COOH}$-terminated SAM to be greater than the

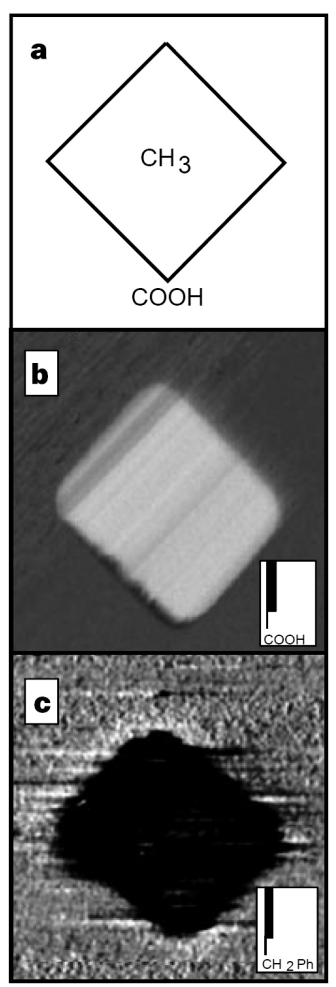

Figure 2. Chemically sensitive imaging with functionalized nanotube tips. a, Diagram of a patterned sample prepared by microcontact printing $^{19}$ and consisting of $10-\mu \mathrm{m}$ squares of a methyl-terminated (hexadecanethiol) SAM region surrounded by a carboxylic acid-terminated (16-mercaptohexadecanoic acid) SAM background on gold. Tapping mode phase-lag images of the patterned sample in ethanol were recorded with $\mathrm{b}$, an unmodified nanotube tip ( $\mathrm{COOH}$-terminated) and $\mathrm{c}$, a benzylamine-functionalized nanotube tip (phenyl terminated). Darker regions indicate greater phase lag; the contrast in $\mathrm{b}$ and $\mathrm{c}$ corresponds to phase variations of $2.3^{\circ}$ and $2^{\circ}$, respectively. The images are $16 \mu \mathrm{m}$ $\times 16 \mu \mathrm{m}$. Images and force curves were acquired with a Nanoscope III (Digital Instruments, Inc.). Imaging parameters were optimized for individual tips; typical ranges for the FESP (force modulation etched silicon probe) nanotube tips were (1) resonant frequencies, $28-33 \mathrm{kHz}$; (2) free r.m.s. oscillation amplitude, $30-90 \mathrm{~nm}$, (3) set-point, 1-3 V, and (4) scan rate, $0.5-1.2 \mathrm{~Hz}$. 
$\mathrm{CH}_{3}$-terminated region, these results are consistent with chemically sensitive imaging. Furthermore, when tips are covalently modified with benzylamine, which produces a hydrophobic tip that interacts more strongly with the $\mathrm{CH}_{3}$ than with the $\mathrm{COOH}$ regions of the sample, the phase contrast is reversed (Figure $2 \mathrm{c}$ ) as expected on the basis of the change in intermolecular interactions. In addition, control experiments carried out using the same modification procedures but without the EDC coupling reagent (see Figure 1 legend) required for
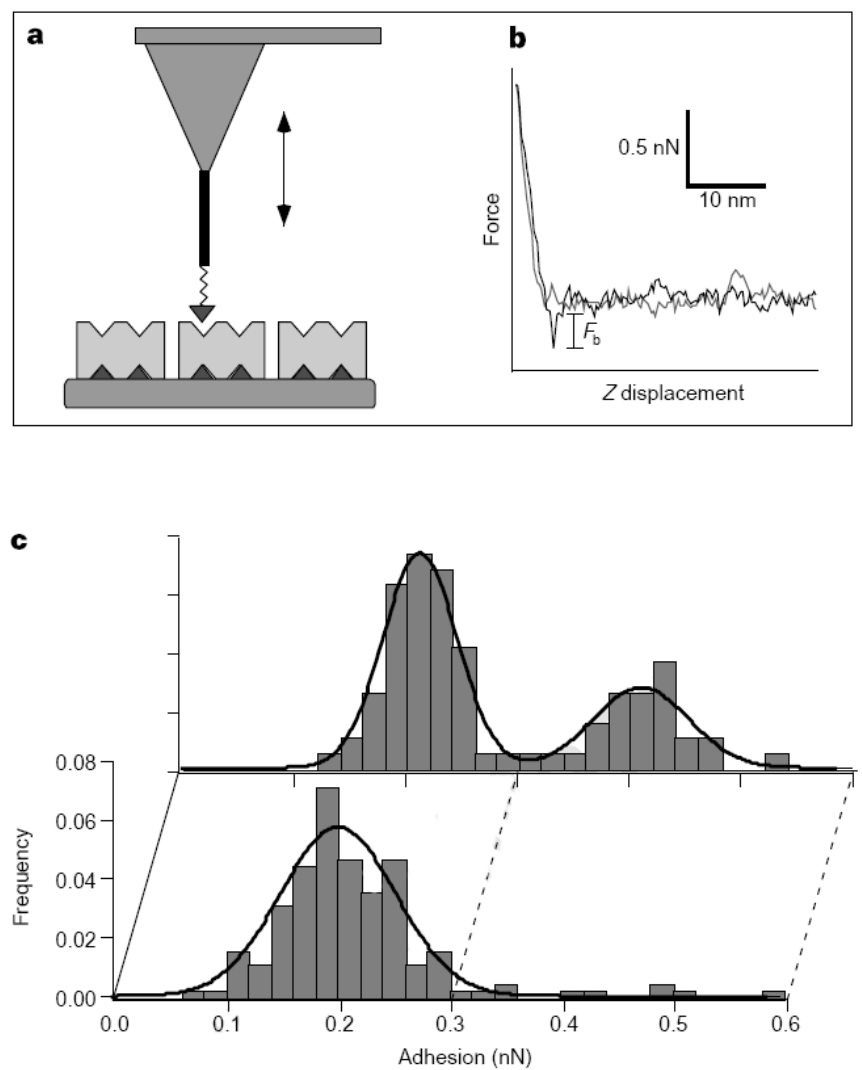

Figure 3. Ligand-derivatized nanotube tips as biological probes, a, Diagram illustrating a nanotube tip covalently modified with a biotin ligand (dark-grey triangle) interacting with streptavidin protein receptors (light-grey blocks); the streptavidin receptors are linked to the surface by biotin groups. The nanotube tips were modified by immersion into a solution containing $5 \mathrm{mM}$ 5-(biotinamido)pentylamine (Pierce), $50 \mathrm{mM}$ EDC and $0.1 \mathrm{M} \mathrm{MES}$ at $\mathrm{pH} 6.0$ for $2 \mathrm{~h}$. The streptavidin protein surface was formed by coating a cleaved mica substrate with $250 \mathrm{\mu g} \mathrm{ml}^{-1}$ biotinamidocaproyl-labelled bovine serum albumin (Sigma) in phosphate buffer saline (PBS) pH 5.6 for $2 \mathrm{~h}$, rinsing in $\mathrm{pH} 7.0 \mathrm{PBS}$, and then incubating with $30 \mathrm{\mu g} \mathrm{ml}^{-1}$ streptavidin (Sigma) in pH 7.0 PBS for two hours. b, Representative force-displacement curve recorded with a biotin-modified nanotube tip on thestreptavidin surface in $\mathrm{pH} 7.0$ PBS. The binding force is indicated by $F_{\mathrm{b}}$. c, Adhesion histograms representing 200-400 force-displacement curves obtained at a repetition rate of $3 \mathrm{~Hz}$ from two separate biotinylated nanotube tips on streptavidin-derivatized surfaces. One tip showed a single peak centred at $200 \mathrm{pN}$ that corresponds to the unbinding of a single biotin-streptavidin ligand-receptor complex. The other tip exhibited a bimodal distribution peaking at 200 and $400 \mathrm{pN}$ that corresponds to the unbinding of one and two biotin-streptavidin ligand-receptor complexes, respectively. The first tip showed single binding events in $36 \%$ of the measurements and no detectable binding in the remaining $64 \%$ of the curves. The second tip showed single and double binding events in $30 \%$ and $15 \%$ of the measurements, respectively. No interaction was detected within experimental error in the remaining $55 \%$ of the data. The frequency was normalized by the total number of force-displacement curves. covalent bond formation show the same phase contrast as the starting tips. These imaging results demonstrate that direct covalent coupling reactions on nanotube tips, which we believe provide a more flexible and robust method of modification than SAMs, can be used to create chemically sensitive imaging probes. The resolution that we achieve in our experiments (Figure 2) is limited by the technique used to create the patterned substrate. The multi-walled nanotubes used here can have diameters of $15-50 \mathrm{~nm}$, but we have recently demonstrated ${ }^{25}$ that lateral resolution of $<3 \mathrm{~nm}$ can be achieved by using $\mathrm{COOH}$-terminated single-walled nanotube ${ }^{21}$ tips on mixed monolayer/bilayer substrates.

Covalently modified nanotube tips also offer the possibility of probing biological systems at the nanometre scale. To illustrate this point we have studied the well characterized ligand-receptor interaction of biotin-streptavidin ${ }^{22}$. 5-(biotinamido)pentylamine was covalently linked to nanotube tips by the formation of an amide bond (Figure 3a). Force-displacement measurements (Figure 3b) made on mica surfaces containing immobilized streptavidin show well defined binding force quanta of $\sim 200 \mathrm{pN}$ per biotin-steptavidin pair (Figure 3c). Control experiments carried out with an excess of free biotin (which blocks all receptor sites of the protein) in solution, and with unmodified nanotube tips showed no adhesion within the noise limits of our experiments, and thus confirm that the observed binding force results from the interaction of nanotube-linked biotin with surface streptavidin. The functionalized nanotube tips usually show only single binding events of $200 \mathrm{pN}$, although with some tips it is also possible to observe events of twice this force; we attribute such events to the simultaneous binding of two biotin-streptavidin pairs. Our measured binding force quanta agree with previous AFM studies in which biotin or avidin were attached to probe tips by the non-specific adsorption of bovine serum albumin ${ }^{23},{ }^{24}$. We believe that our results show that it will be possible (by using well defined covalent chemistry) to attach individual active ligands, proteins or other macromolecules in a spatially defined manner to the ends of nanotubes, and then to use these functionalized probes to create high-resolution maps of binding domains on, for example, proteins and membranes. Such experiments would be difficult using conventional tips modified either using nonspecific adsorption or with SAMs.

The covalent modification of nanotube tips enables the straightforward creation of well defined probes which are sensitive to specific intermolecular interactions that define the properties of many chemical and biological systems. In addition to the directions indicated above, we believe that functionalized nanotube tips will prove especially useful for imaging self-assembled polymeric and biological materials. In particular, recent studies in which we have extended the covalent modification procedures to single-walled nanotubes ${ }^{25}$ suggest the possibility of mapping functional groups with true molecular resolution. Among intriguing future applications is the use of the highly selective and robust chemistry described here to link catalysts, such as transition-metal complexes, to nanotube ends to create tools that could modify or create structures at the molecular scale. The selective functionalization of nanotube ends might also open up the possibilities of creating interconnections for electronic devices on a nanometre scale and assembling new classes of materials from nanotubes.

\section{References}

1. Binnig, G., Quate, C. F. \& Gerber, C. Atomic force microscope. Phys. Rev. Lett. 56, 930-933 (1986).

2. Hansma, P. K. et al. Tapping mode atomic force microscopy in liquids. Appl. Phys. Lett. 64, 1738-1740 (1994).

3. Bustamante, C. , Rivetti, C. \& Keller, D. J. Scanning force microscopy under aqueous solutions. Curr. Opin. Struct. Biol. 7, 709-716 (1997).

4. Dai, H. , Hafner, J. H. , Rinzler, A. G., Colbert, D. T. \& Smalley, R. E. 
Nanotubes as nanoprobes in scanning probe microscopy. Nature $\mathbf{3 8 4}$, $147-150$ (1996).

5. Wong, S. S. , Harper, J. D. , Lansbury, P. T. \& Lieber, C. M. Carbon nanotube tips: high-resolution probes for imaging biological systems. J. Am. Chem. Soc. 120, 603-604 (1998).

6. Wong, E. W. , Sheehan, P. E. \& Lieber, C. M. Nanobeam mechanisms: elasticity, strength and toughness of nanorods and nanotubes. Science 277, 1971-1975 (1997).

7. Keller, D. Ananotube molecular tool. Nature 384, 111 (1996).

8. March, J. Advanced Organic Chemistry (Wiley, New York, 1992).

9. Bodanszky, M. \& Bodanszky, A. The Practice of Peptide Synthesis 2nd edn (Springer, New York, 1994).

10. Hiura, H. , Ebbesen, T. W. \& Tanigaki, K. Opening and purification of carbon nanotubes in high yields. Adv. Mater. 7, 275-276 (1995).

11. Evans, J. F. \& Kuwant, T. Electrocatalysis of solution species using modified electrodes. J. Electroanal. Chem. 80, 409-416 (1977).

12. Wandass, J. H. , Gardella, J. A., Weinberg, N. L. , Bolster, M. E. \& Salvati, L. X-ray photoelectron and scanning Auger electron spectroscopic studies of oxidized graphite electrode surfaces. J. Electrochem. Soc. 134, 2734-2739 (1987).

13. Frisbie, C. D. , Rozsnyai, L. F. , Noy, A., Wrighton, M. S. \& Lieber, C. M. Functional group imaging by chemical force microscopy. Science $\mathbf{2 6 5}$, 2071-2074 (1994).

14. Noy, A., Vezenov, D. V. \& Lieber, C. M. Functional force microscopy. Annu. Rev. Mater. Sci. 27, 381-421 (1997).

15. McKendry, R., Theoclitou, M.-E., Rayment, T. \& Abell, C. Chiral discrimination by chemical force microscopy. Nature 391, 566-568 (1998).

16. Vezenov, D. V. , Noy, A., Rozsnyai, L. F. \& Lieber, C. M. Force titrations and ionization state imaging of functional groups in aqueous solutions by chemical force microscopy. J. Am. Chem. Soc. 119, 2006-2015 (1997).

17. Marti, A. , Hahner, G. \& Spencer, N. D. Sensitivity of frictional forces to $\mathrm{pH}$ on a nanometer scale: a lateral force microscopy study. Langmuir 11, 4632-4635 (1995).
18. Giles, M. A., Hudson, A. Q. \& Borders, C. L. J Stability of water-soluble carbodiimides in aqueous solution. Anal. Biochem. 184, 244-248 (1990).

19. Kumar, A. , Biebuyck, H. \& Whitesides, G. M. Patterning self-assembled monolayers: applications in materials science. Langmuir 10, 1498-1511 (1994).

20. Noy, A., Sanders, C. H. , Vezenov, D. V. , Wong, S. S. \& Lieber, C. M. Chemically-sensitive imaging in tapping mode by chemical force microscopy: relationship between phase lag and adhesion. Langmuir 14, 15081511 (1998).

21. Thess, A. et al. Crystalline ropes of metallic carbon nanotubes. Science 273, 483-487 (1996).

22. Livnah, O., Bayer, E. A., Wilchek, M. \& Sussman, J. L. Three-dimensional structures of avidin and the avidin-biotin complex. Proc. Natl Acad. Sci. USA 90, 5076-5080 (1993).

23. Florin, E.-L. , Moy, V. T. \& Gaub, H. E. Adhesion forces between individual ligand-receptor pairs. Science 264, 415-417 (1994).

24. Lee, G. U. , Kidwell, D. A. \& Colton, R. J. Sensing discrete streptavidinbiotin interactions with atomic force microscopy. Langmuir 10, 354-357 (1994).

25. Wong, S. S., Woolley, A. T. , Joselevich, E., Cheung, C. L. \& Lieber, C. M. Covalently-functionalized single-walled carbon nanotube probe tips for chemical force microscopy. J. Am. Chem. Soc. (submitted).

\section{Acknowledgements}

We thank D. Vezenov and A. Noy for discussions. C.M.L. was supported by the US Air Force Office of Scientific Research. S.S.W., E.J. and A.T.W. acknowledge fellowships from the Natural Sciences and Engineering Research Council of Canada, the Hebrew University of Jerusalem, and the Damon Runyon-Walter Winchell Foundation, respectively. 